\section{The Metabolic Syndrome and Male Infertility: A Review of the Literature}

\author{
Caner EDIZ and Ramazan ALTINTAS* \\ Medicine Faculty, Urology Department, Inonu University, Malatya, Turkey
}

\begin{abstract}
The Metabolic Syndrome (MetS) is a constellation of various risk factors, including obesity, dyslipidemia, hypertension, and impaired glucose metabolism, with insulin resistance as the hypothesized underlying pathogenic mechanism. In recent years, the Metabolic Syndrome (MetS) is note worthy due to its association with the development of cardiovascular disease and type 2 diabetes mellitus. According to the American Heart Association, 47 million Americans have MetS. The numerous deleterious effects of MetS are being investigated throughout the medical community, as MetS may potentially affect many aspects of human physiology due to its systemic nature. The impaired glucose metabolism is widely accepted as a negative effecting factor on human fertility in either gender. Obesity appears to be associated with male reproductive dysfunction and infertility. Elevated Free Fatty Acids (FFAs), associated with dyslipidemia in MetS, can cause endothelial dysfunction through increased free radical production and inhibition of NO synthesis.

Infertility, defined as the inability to conceive after one year of unprotected intercourse, affects approximately $15 \%$ of couples. Male factor infertility is the sole cause of infertility in approximately $20 \%$ of infertile couples, with an additional $30 \%$ to $40 \%$ secondary to both male and female factors. Thus, male factor infertility is present in approximately half of all infertile couples. Recently in some studies, it has been reported that increased body mass index negatively affects on male fertility or semen parameters. This review will evaluate MetS and its components in order to establish a paradigm with male factor infertility.
\end{abstract}

Keywords: Diabates mellitus; Dyslipidemia; Hypertension; Male infertility; Metabolic syndrome; Obesity

*Corresponding author: Ramazan ALTINTAS, Medicine Faculty, Urology Department, Inonu University, Malatya, Turkey, Tel: +9042234106605804; Fax: 04223410728; E-mail: ramazan.altintas@inonu.edu.tr

Citation: Ediz C, Altintas R (2014) The Metabolic Syndrome and Male Infertility: A Review of the Literature. J Diabetes Metab Disord 1: 002.

Received: June 29, 2014; Accepted: November 03, 2014; Published: November 17, 2014

Copyright: (c) 2014 Ediz C and Altintas R, This is an open-access article distributed under the terms of the Creative Commons Attribution License, which permits unrestricted use, distribution, and reproduction in any medium, provided the original author and source are credited.

\section{Introduction}

The "Metabolic Syndrome" (MetS) is a clustering of components that reflect overnutrition, sedentary lifestyles and resultant excess adiposity. The MetS includes the clustering of abdominal obesity, insulin resistance, dyslipidemia and elevated blood pressure and is associated with other comorbidities including the prothrombotic state, proinflammatory state, nonalcoholic fatty liver disease and reproductive disorders. The impaired glucose metabolism is widely accepted as a negative effecting factor on human fertility in either gender. Obesity appears to be associated with male reproductive dysfunction and infertility. Elevated Free Fatty Acids (FFAs), associated with dyslipidemia in MetS, can cause endothelial dysfunction through increased free radical production and inhibition of NO synthesis. We aim to discuss summarize current evidence in the infertile male patients with MetS in the literature.

\section{Obesity and Infertility}

In Western countries, people are considered obese when their Body Mass Index (BMI) a measurement obtained by dividing a person's weight by the square of the person's height, exceeds $30 \mathrm{~kg} / \mathrm{m}^{2}$, with the range $25-30 \mathrm{~kg} / \mathrm{m}^{2}$ defined as overweight. Obesity is becoming an increasingly common cause for infertility and it is a cardinal feature of MetS. Adverse effects of obesity on male fertility are postulated to occur through several mechanisms. First, peripheral conversion of testosterone to estrogen in excess peripheral adipose tissue may lead to secondary hypogonadism through hypothalamic-pituitarygonadal axis inhibition. Second, oxidative stress at the level of the testicular micro environment may result in decreased spermatogenesis and sperm damage. Lastly, the accumulation of supra pubicand inner thigh fat may result in increased scrotal temperatures in severely obese men [1].

Multiple explanations have been proposed for the decline in male fertility including increased prevalence of obesity and exposure to environmental pollution during fetal or adult life. Hedley et al., estimated the current prevalence of obesity at $30.6 \%$ [2].

Multiple reports have described the effects of obesity on male fertility [3-5]. Few studies have demonstrated perturbation in the hypothalamic-pituitary-gonadal axis in obese men with resultant significant decrease in sex hormone-binding globulin and total testosterone [6-11]. Variable results were noted for free testosterone in the various studies cited. Men with obesity, the metabolic syndrome and type 2 diabetes have low total and free testosterone and low Sex Hormone-Binding Globulin (SHBG). Conversely, the presence of low testosterone and/or SHBG predicts the development of metabolic syndrome and type 2 diabetes [12]. Thus, the observed decrease in testosterone levels in obese males is likely due to several factors, including decreased synthesis of testosterone, inhibition of SHBG synthesis, and decreased gonadotropin secretion [12]. In summary, total testosterone, free testosterone and SHBG are all commonly decreased in obese males. In the studies, Follicle-Stimulating Hormone (FSH) and Luteinizing Hormone (LH) levels were normal or low in obese men $[6,7,10]$. 
Aromatase is important in men's health, obesity, the metabolic syndrome, type 2 diabetes and aging. In males with increasing obesity there is increased aromatase activity, which irreversibly converts testosterone to estradiol resulting in decreased testosterone and elevated estrogen levels [13]. Elevated estrogens, made by aromatization of androgens in peripheral adipose tissue in obese men, provide a possible explanation for suppression of the hypothalamic-pituitary axis $[9,10]$.

Interestingly, infertile/obese males had significantly lower SHBG with significantly higher levels of bioavailable testosterone and estradiol. In the studies further highlights the need for additional evidence correlating hormonal dysregulation in obese males to infertility. As a result, this population may be at an increased risk for infertility.

Obesity was defined as greater than 135\% of ideal bodyweight, not by BMI. Jarow and colleagues studied 120 men categorized into obese or nonobese, and fertile or infertile groups. Fertility status was determined by records at an infertility clinic indicating infertile marriages of more than 1 year and abnormal semen parameters. The authors found that men who were both infertile and obese had significantly lower testosterone levels and testosterone-estradiol ratios than fertile/nonobese, fertile/obese, and infertile/nonobese counterparts [14].

To date, few studies have examined the relationship between objective measures of obesity and semen quality. Magnusdottir et al., examined lifestyle and environmental factors, which have been hypothesized to adversely affect semen quality. The study population included 72 men from a single clinic for assisted reproduction who had been categorized into 3 groups: Male Factor Subfertility (MFS), Female Factor Subfertility (FFS), and Idiopathic Subfertility (IS), with semen analyzed per World Health Organization (WHO, 1999) criteria. The authors found a threefold increased incidence of obesity (BMI $>30$ $\mathrm{kg} / \mathrm{m}^{2}$ ) in patients with MFS compared with the other 2 groups. Furthermore, patients in the MFS group had a significantly higher BMI (27.8 [range, 21.8-38.6]) compared with those in the FFS group (25 [range, 19.7-45.6]). The authors also examined levels of work activity, defined as sedentary (office environment), intermediate (salesmen), andactive (laborers). They found that when patients in the IS and FFS groups were stratified into groupings of "low normal" and "high normal" sperm concentrations, significantly more men with low normal sperm density had an associated sedentary level of work activity [15]. These results were attributed by the authors to elevated scrotal temperatures associated with sedentary activity rather than with obesity itself. Although these results are intriguing, they are limited by sample size and self reported heights and weights.

Obesity appears to be associated with male reproductive dysfunction and infertility, although this has been inconsistent and inconclusive. Insulin and leptin are known mediators and modulators of the hypothalamus-pituitary-testes axis, contributing to the regulation of male reproductive potential and overall wellbeing. These hormones are also present in semen influencing sperm functions. Although abdominal obesity is closely associated with insulin resistance (hyperinsulinaemia), hyperleptinaemia and glucose dysfunction, changes in seminal plasma concentrations of insulin, leptin and glucose in obese males has not previously been investigated. Obesity was associated with increased serum and seminal insulin and leptin, with no significant difference in seminal glucose. Hyperinsulinaemia and hyperleptinemia are associated with increased seminal insulin and leptin concentrations, which may negatively impact male reproductive function in obesity [16].

Eisenberg ML et al., showed the relationship between semen parameters and BMI. When examining semen parameters, ejaculate volume showed a linear decline with increasing BMI and Waist Circumference (WC). Similarly, the total sperm count showed a negative linear association with WC. No significant relationship was seen between body size (i.e. BMI or WC) and semen concentration, motility, vitality, morphology or DNA fragmentation index. Overweight and obesity are associated with a higher prevalence of low ejaculate volume, sperm concentration and total sperm count [17].

Smoking, diabetes and obesity are independently associated with increased oxidative stress in men. Oxidative stress is a pathophysiologic process commonin a number of disease states, including autoimmune, cardiovascular and infectious processes. Oxidative stress arises when an excess concentration of Reactive Oxidative Species (ROS), molecules harboringan unpaired electron, are present in a particular physiologic environment. These highly reactive and unstable molecules are capable of inducing significant cellular damage throughout the body. With regard to male reproductive health, several studies have revealed that oxidative stress results in sperm membrane lipid peroxidation with impairment in sperm motility and sperm-oocyte interaction. Kodama and colleagues showed that the DNA of spermatozoa from infertile men had greater oxidative injury when compared to controls [18]. In summary, oxidative stress may also result in lipid peroxidation of the sperm membrane, leading to decreased motility and membrane dysfunction; excessive oxidative stress may also result in sperm DNA damage, with diminished genetic viability of the affected sperm.

\section{Diabetes and Infertility}

Diabetes Mellitus (DM), associated with very subtle disorders, affects, either directly or indirectly, various functions of the reproductive system. Insulin resistance is considered by many investigators to be an underlying pathologic aberration in MetS.

The data available suggest that DM alters conventional sperm parameters. In addition, DM causes histologic damage of the epididymis, with a negative impact on sperm transit. Various mechanisms may explain the sperm damage observed in patients with DM. These include endocrine disorders, neuropathy, and increased oxidative stress. Many authors suggest that DM decreases serum testosterone levels. This is associated with a steroidogenetic defect in Leydig cells. In addition, diabetic neuropathy seems to cause atonia of seminal vesicles, bladder, and urethra. Furthermore, DM is associated with an increased oxidative stress, which damages sperm nuclear and mitochondrial DNA [19].

A growing body of literature has detailed the relationship between hypogonadism and type 2 diabetes mellitus including 3 epidemiological studies that suggest hypogonadismis a risk factor for diabetes [20-22].

Ali and associates evaluated 314 men with NIDDM and neuropathy, they reported a higher sperm concentration and lower sperm motility compared with diabetic men without neuropathy and controls [23]. Though limited, these data suggest sperm dysfunction in some men with NIDDM. The factors leading to paradoxically increased $s$ perm concentration in men with NIDDM and neuropathy in this study are unclear, but the authors observed a decrease in semen 
volume in these patients, which may suggest decreased seminal secretion and an overall concentration of the ejaculated sperm.

Erectile Dysfunction (ED), failure of seminal emission, and retrograde ejaculation are known complications of NIDDM that have an impact on male reproductive potential. ED in patients with NIDDM stems in part from autonomic neuropathy and vascular disease. Failure of emission and retrograde ejaculation also result from autonomic neuropathy, with an estimated $32 \%$ of men with DM affected by some degree of ejaculatory dysfunction [24]. In total, ejaculatory dysfunction may represent the mostcommon cause of infertility in diabetic men [25].

\section{Dyslipidemia and Infertility}

Dyslipidemia is another sentinel feature of MetS that may have an impact on semen quality and fertility. Kasturi and colleagues proposed that dyslipidemia and a proinflammatory state may result in increased Reactive Oxygen Species (ROS) formation and subsequent lipid peroxidation of the spermatic membranes [26].

Ramirez-Torres and colleagues studied 106 malepartners from infertile couples, reporting a $65 \%$ incidence of dyslipidemia as defined by isolated hypercholesterolemia, triglyceridemia or both. The incidenceof obesity (18\%), overweight (30.2\%), hypertension( $26 \%)$, glucose intolerance (15\%) and DM (4.7\%) were also reported, although no correlation with sperm abnormalities was observed [27]. While this study suggests a relationship between lipid abnormalities and infertility, no clear mechanism was postulated. However, as described previously, oxidative stress is an attractive candidate.

This hypothesis is supported in a former study by Shalaby and associates [28]. The authors examined the effects of a high-cholesterol diet and anti cholesterol therapy on male rat fertility, finding that male rats fedwith a high-cholesterol diet ( $1 \%$ by composition) had significant declines in fertility, testicular weight and sperm characteristics compared with male rats with acholesterol-free diet. Treatment with a-tocopherol, simvastatin and the two in combination significantly increased the fertility index (mating success rate) from $42.5 \%$ to $71.5 \%, 61.25 \%$, and $79.5 \%$, respectively. Additionally, all three treatment groups demonstrated significantly increased testicular weight, sperm count, sperm motility, sperm viability, and significantly decreased sperm abnormalities.

\section{Hypertension and Infertility}

Hypertension (HTN) is defined as a blood pressure greater than $130 / 85 \mathrm{mmHg}$ by ATP III criteria, and it represents a major risk factor for cardiovascular disease. While hypertension is a well-established risk factor for $\mathrm{ED}$, the direct effect on male fertility, if any, is not as well understood. End-organ damage is a well-documented aspect of hypertension, but to date, testicular end-organ injury caused by HTN has not been clearly defined. Several studies examining hypertensive men demonstrated a significant inverse relationship between blood pressure and total serum testosterone, which could be associated with impaired reproductive potential [29-31], free testosterone [32-34] and SHBG [34].

\section{Oxidative Stress and Infertility}

Oxidative stress plays a pathological role in the development of various diseases including diabetes, atherosclerosis, or infertility. Systemic oxidative stress results from an imbalance between oxidants derivatives production and antioxidants defenses. Obesity dramatically increases the risk of development of metabolic disease. This risk appears to originate from disruption in adipose tissue function leading to a chronic inflammatory state. A systemic pro-inflammatory state in combination with hyperglycemia, insulin resistance, oxidative stress and activation of the renin angiotensin system are systemic disturbances in obese individuals that contribute independently and synergistically to decreasing NO bioavailability [35]. Thus, the chronic inflammation can be caused to damage in male reproductive system. Also in healthy subjects, infusion of FFA causes increased oxidative stress and insulin resistance, which can be reversed by infusion with antioxidants such as glutathion [36]. Oxidative stress has been identified as one of the many mediators of male infertility by causing sperm dysfunction.

\section{Conclusion}

MetS is a complex disorder consisting of numerous interrelated pathophysiologic entities including obesity, HTN, dyslipidemia, hyperglycemia/IR, and endothelial dysfunction. Male infertility may represent in some patients with MetS. Obesity/overweight may result in increased scrotal temperatures, impaired spermatogenesis, decreased sperm concentration and motility, increased sperm DNA damage and hypogonadism. Similarly, type 2 diabetes mellitus may contribute to and compound this continuity. Dyslipidemia with increased oxidative stress in the testicular microenvironment may further decrease fertility. Additional studies are needed to fully clarify the pathophysiologic link and evidence between the components of MetS and male infertility.

\section{Acknowledgments}

The authors thank for your technical help and writing assistance.

\section{Disclosure}

The authors report no conflicts of interest in this work

\section{References}

1. Stokes VJ, Anderson RA, George JT (2014) How does obesity affect fertility in men - and what are the treatment options? Clin Endocrinol (Oxf).

2. Hedley AA, Ogden CL, Johnson CL, Carroll MD, Curtin LR, et al. (2004) Prevalence of overweight and obesity among US children, adolescents, and adults, 1999-2002. JAMA 291: 2847-2850.

3. Hammoud AO, Gibson M, Peterson CM, Hamilton BD, Carrell DT (2006) Obesity and male reproductive potential. J Androl 27: 619-626.

4. Hammoud AO, Gibson M, Peterson CM, Meikle AW, Carrell DT (2008) Impact of male obesity on infertility: a critical review of the current literature. Fertil Steril 90: 897-904.

5. MacDonald AA, Herbison GP, Showell M, Farquhar CM (2010) The impact of body mass index on semen parameters and reproductive hormones in human males: a systematic review with meta-analysis. Hum Reprod Update 16: 293-311.

6. Glass AR, Swerdloff RS, Bray GA, Dahms WT, Atkinson RL (1977) Low serum testosterone and sex-hormone-binding-globulin in massively obese men. $\mathrm{J}$ Clin Endocrinol Metab 45: 1211-1219.

7. Amatruda JM, Harman SM, Pourmotabbed G, Lockwood DH (1978) Depressed plasma testosterone and fractional binding of testosterone in obese males. J Clin Endocrinol Metab 47: 268-271.

8. Schneider G, Kirschner MA, Berkowitz R, Ertel NH (1979) Increased estrogen production in obese men. J Clin Endocrinol Metab 48: 633-638.

9. Kley HK, Deselaers T, Peerenboom H, Krüskemper HL (1980) Enhanced conversion of androstenedione to estrogens in obese males. J Clin Endocrinol Metab 51: 1128-1132. 
10. Strain GW, Zumoff B, Kream J, Strain JJ, Deucher R, et al. (1982) Mild Hypogonadotropic hypogonadism in obese men. Metabolism 31: 871-875.

11. Zumoff B, Strain GW, Miller LK, Rosner W, Senie R, et al. (1990) Plasma free and non-sex-hormone-binding-globulin-bound testosterone are decreased in obese men in proportion to their degree of obesity. J Clin Endocrinol Metab 71: 929-931.

12. Pasquali R, Casimirri F, De lasio R, Mesini P, Boschi S, et al. (1995) Insulin regulates testosterone and sex hormone-binding globulin concentrations in adult normal weight and obese men. J Clin Endocrinol Metab 80: 654-658.

13. Cohen PG (2008) Obesity in men: the hypogonadal-estrogen receptor relationship and its effect on glucose homeostasis. Med Hypotheses 70: 358-360.

14. Jarow JP, Kirkland J, Koritnik DR, Cefalu WT (1993) Effect of obesity and fertility status on sex steroid levels in men. Urology 42: 171-174

15. Magnusdottir EV, Thorsteinsson T, Thorsteinsdottir S, Heimisdottir M, Olafs dottir K (2005) Persistent organochlorines, sedentary occupation, obesity and human male subfertility. Hum Reprod 20: 208-215.

16. Leisegang K, Bouic PJ, Menkveld R, Henkel RR (2014) Obesity is associated with increased seminal insulin and leptin alongside reduced fertility parameters in a controlled male cohort. Reprod Biol Endocrinol 12: 34

17. Eisenberg ML, Kim S, Chen Z, Sundaram R, Schisterman EF, et al. (2014) The relationship between male BMI and waist circumference on semen quality: data from the LIFE study. Hum Reprod 29: 193-200.

18. Kodama H, Yamaguchi R, Fukuda J, Kasai H, Tanaka T (1997) Increased oxidative deoxyribonucleic acid damage in the spermatozoa of infertile male patients. Fertil Steril 68: 519-524.

19. La Vignera S, Condorelli R, Vicari E, D’Agata R, Calogero AE (2012) Diabetes mellitus and sperm parameters. J Androl 33: 145-153.

20. Haffner SM, Shaten J, Stern MP, Smith GD, Kuller L (1996) Low levels of sex hormone-binding globulin and testosterone predict the development of non-insulin-dependent diabetes mellitus in men. MRFIT Research Group. Multiple Risk Factor Intervention Trial. Am J Epidemiol 143: 889-897.

21. Stellato RK, Feldman HA, Hamdy O, Horton ES, McKinlay JB (2000) Testosterone, sex hormone-binding globulin, and the development of type 2 diabetes in middle-aged men: prospective results from the Massachusetts male aging study. Diabetes Care 23: 490-494.

22. Oh JY, Barrett-Connor E, Wedick NM, Wingard DL, Rancho Bernardo Study (2002) Endogenous sex hormones and the development of type 2 diabetes in older men and women: the Rancho Bernardo study. Diabetes Care 25: 55-60.
23. Ali ST, Shaikh RN, Siddiqi NA, Siddiqi PQ (1993) Semen analysis in insulindependent/non-insulin-dependent diabetic men with/without neuropathy. Arch Androl 30: 47-54.

24. Shaban S, Seaman E, Lipschultz LI (1991) Treatment of abnormalities of ejaculation. In: Lipschultz LI, Howards SS (eds.). Infertility in the Male. (3rdedn), St. Louis: Mosby Year Book, USA. Pg no: 423-438.

25. Sexton WJ, Jarow JP (1997) Effect of diabetes mellitus upon male reproductive function. Urology 49: 508-513.

26. Kasturi SS, Tannir J, Brannigan RE (2008) The metabolic syndrome and male infertility. J Androl 29: 251-259.

27. Ramírez-Torres MA, Carrera A, Zambrana M (2000) [High incidence of hyperestrogenemia and dyslipidemia in a group of infertile men]. Ginecol Obstet Mex 68: 224-229.

28. Shalaby MA, el-Zorba HY, Kamel GM (2004) Effect of alpha-tocopherol and simvastatin on male fertility in hypercholesterolemic rats. Pharmacol Res 50: 137-142.

29. Barrett-Connor E, Khaw KT, Yen SS (1990) Endogenous sex hormone levels in older adult men with diabetes mellitus. Am J Epidemiol 132: 895-901.

30. Phillips GB (1993) Relationship between serum sex hormones and the glucose-insulin-lipid defect in men with obesity. Metabolism 42: 116-120.

31. Fogari R, Preti P, Derosa G, Marasi G, Zoppi A, et al. (2002) Effect of antihypertensive treatment with valsartan or atenolol on sexual activity and plasma testosterone in hypertensive men. Eur J Clin Pharmacol 58: 177-180.

32. Hughes GS, Mathur RS, Margolius HS (1989) Sex steroid hormones are altered in essential hypertension. J Hypertens 7: 181-187.

33. Phillips GB, Jing TY, Resnick LM, Barbagallo M, Laragh JH, et al. (1993) Sex hormones and hemostatic risk factors for coronary heart disease in men with hypertension. J Hypertens 11: 699-702.

34. Svartberg J, von Mühlen D, Schirmer H, Barrett-Connor E, Sundfjord J, et al. (2004) Association of endogenous testosterone with blood pressure and left ventricular mass in men. The Tromsø Study. Eur J Endocrinol 150: 65-71.

35. Iantorno M, Campia U, Di Daniele N, Nistico S, Forleo GB, et al. (2014) Obesity, inflammation and endothelial dysfunction. J Biol Regul Homeost Agents 28: $169-176$.

36. Le Lay S, Simard G, Martinez MC, Andriantsitohaina R (2014) Oxidative stress and metabolic pathologies: from an adipocentric point of view. Oxid Med Cell Longev 2014: 908539. 